REVISTA PORTUGUESA DE PSICOLOGIA

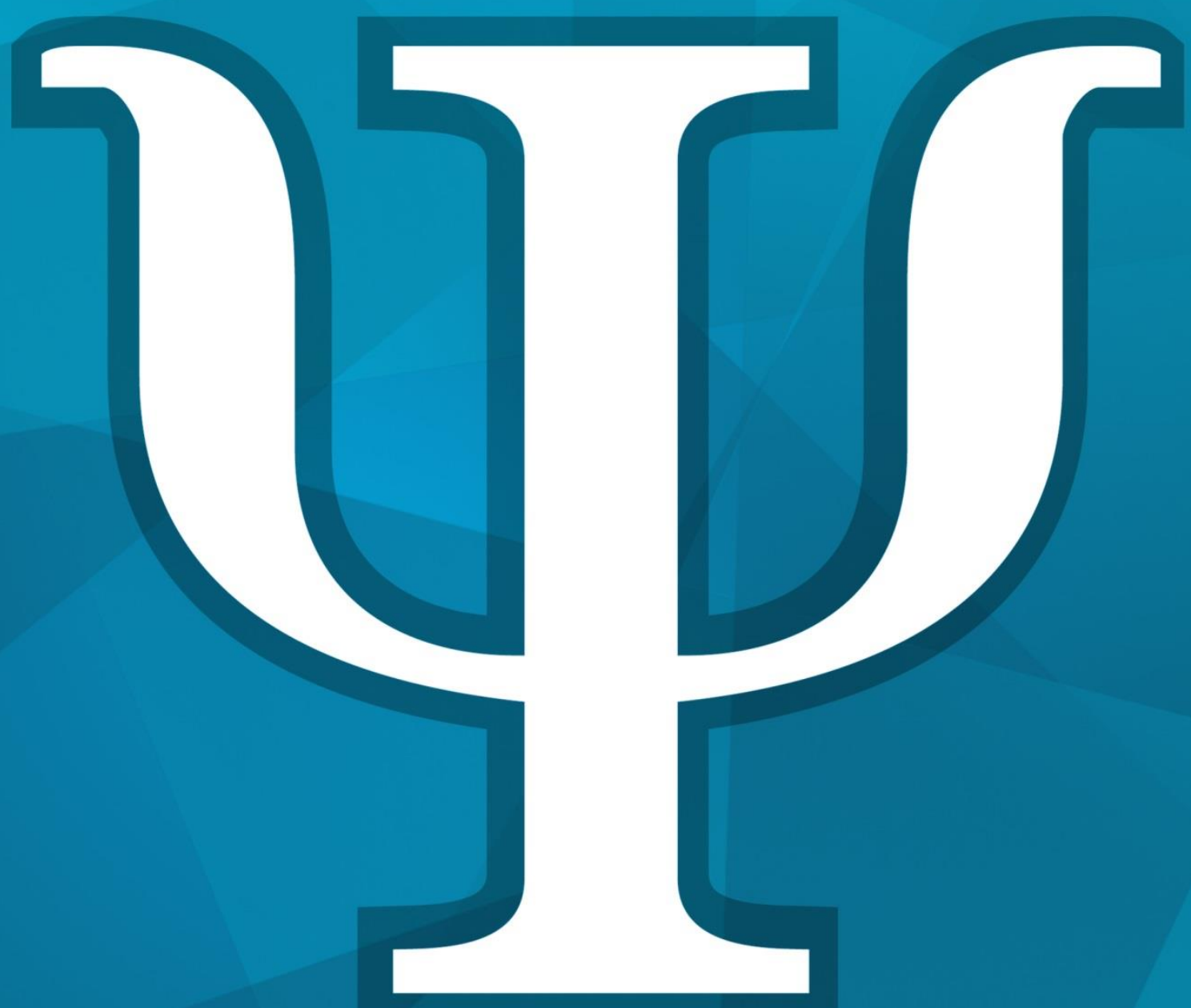





\section{Conselho Editorial / Editorial Board}

\section{Editor:}

João Manuel Moreira

Faculdade de Psicologia, Universidade de Lisboa

\section{Conselho Editorial / Editorial Board:}

Adelina Lopes da Silva

Faculdade de Psicologia, Universidade de Lisboa

Alexandra Reis

Faculdade de Ciências Humanas e Sociais, Universidade do Algarve

Amâncio da Costa Pinto

Faculdade de Psicologia e CE, Universidade do Porto

Ana Margarida Veiga Simão

Faculdade de Psicologia, Universidade de Lisboa

Bárbara Figueiredo

Escola de Psicologia, Universidade do Minho

Danilo Silva

Faculdade de Psicologia, Universidade de Lisboa

Félix Neto

Faculdade de Psicologia e CE, Universidade do Porto

Helio Carpinteiro

Universidade Complutense de Madrid

Isabel Sá

Faculdade de Psicologia, Universidade de Lisboa

Isabel Soares

Escola de Psicologia, Universidade do Minho

Jean Guichard

Conservatoire National des Artes et Métiers

José Frederico Marques

Faculdade de Psicologia, Universidade de Lisboa

José H. Ferreira-Marques

Faculdade de Psicologia, Universidade de Lisboa

José Keating

Escola de Psicologia, Universidade do Minho

José Tomás da Silva

Faculdade de Psicologia e CE, Universidade de Coimbra

Leonel Garcia-Marques

Faculdade de Psicologia, Universidade de Lisboa

Leonor Cardoso

Faculdade de Psicologia e CE, Universidade de Coimbra

Luísa Barros

Faculdade de Psicologia, Universidade de Lisboa

Luísa Morgado

Faculdade de Psicologia e CE, Universidade de Coimbra

Manuel Rafael

Faculdade de Psicologia, Universidade de Lisboa
Maria do Céu Taveira

Escola de Psicologia, Universidade do Minho

Maria Eduarda Duarte

Faculdade de Psicologia, Universidade de Lisboa

Maria José Chambel

Faculdade de Psicologia, Universidade de Lisboa

Mário Ferreira

Faculdade de Psicologia, Universidade de Lisboa

Mário Simões

Faculdade de Psicologia e CE, Universidade de Coimbra

Mark Savickas

Northeast Ohio Medical University

Paulo Ventura

Faculdade de Psicologia, Universidade de Lisboa

Pedro B. Albuquerque

Escola de Psicologia, Universidade do Minho

Rocío Fernandez-Ballesteros

Universidad Autónoma de Madrid

Rosário Lima

Faculdade de Psicologia, Universidade de Lisboa

Salomé Vieira Santos

Faculdade de Psicologia, Universidade de Lisboa

São Luís Castro

Faculdade de Psicologia e CE, Universidade do Porto

\section{Assistente Editorial / Editorial Assistant:}

Rita Monteiro

Editores e Directores Eméritos / Emeriti Editors and Directors:

Henrique Barahona Fernandes

Director, 1967-1991

Agostinho Pereira

Editor, 1967-1969

José Luiz Simões da Fonseca Editor, 1969-1971

José H. Ferreira-Marques

1992-2001

Danilo Silva

2002-2005

Manuel Rafael

2006-2011

\section{Instruções aos autores:}

https://sites.google.com/site/revistaportuguesadepsicologia /instrucoes-aos-autores 



\title{
Reconhecimento de palavras e velocidade de leitura de texto: Um estudo com medidas repetidas no ensino primário.
}

\author{
João Arménio Lopes \\ Universidade do Minho \\ Carminda Filipe Fernandes \\ Universidade do Minho \\ António Vargas Moniz \\ Universidade dos Açores
}

\section{RESUMO}

De entre os diversos processos envolvidos na velocidade com que os sujeitos lêem textos apropriados à idade, o reconhecimento individual de palavras parece desempenhar um papel de especial relevo. Neste trabalho, é apresentado um estudo sobre a relação entre o reconhecimento de palavras isoladas com tipologias específicas (palavras frequentes, palavras raras, pseudopalavras, palavras regulares e palavras irregulares) e a velocidade de leitura de texto em três momentos ao longo do tempo. Participaram no estudo 92 alunos do $1 .^{\circ}$ e do $2 .^{\circ}$ ano de escolaridade. As crianças foram avaliadas no final do $1 .^{\circ}$ ano de escolaridade, a meio do $2 .^{\circ}$ ano de escolaridade e no final do $2 .^{\circ}$ ano. De uma forma geral, os resultados evidenciaram que a velocidade de leitura de palavras isoladas constitui um excelente preditor da velocidade de leitura de texto nos diferentes momentos de avaliação. Contudo, a evidência quanto ao valor preditivo da velocidade de leitura de um tipo específico de palavras relativamente à velocidade da leitura de texto é equívoco. Subsidiariamente, a análise da estabilidade (tracking) das mudanças intraindividuais nas diferenças interindividuais, no que diz respeito à velocidade de leitura de texto, permitiu determinar que os valores iniciais de desempenho na velocidade de leitura de texto condicionam os valores finais da velocidade leitora, mas não para todos os sujeitos.

Palavras-chave: reconhecimento de palavras, velocidade leitora, tipologias de palavras, tracking

\begin{abstract}
Individual word recognition seems to be one of the most important variables involved in appropriate age-level reading speed. This study examines the relation between isolated sets of words (frequent words, infrequent words, pseudowords, regular words and irregular words) reading speed and text reading speed in three different moments in time. Participants were 92 1st and 2nd grade students. Children were tested by the end of 1 st grade, in the middle of 2 nd grade and by the end of 2 nd grade. Overall results show that individual word reading speed is an excellent predictor of text reading speed in the three moments. However, it is not clear whether the reading speed of any particular set of isolated words is a good predictor of text reading speed. The tracking of intraindividual change in interindividual differences also showed that the baseline text reading speed affects the reading speed by the end of second grade, but not for every participant.
\end{abstract}

Keywords: word recognition, reading speed, word typologies, tracking 


\section{INTRODUÇÃO}

É hoje relativamente consensual que a leitura de um texto envolve competências de descodificação, fluência e compreensão leitora, para além de processos de compreensão oral (Burke, Crowder, Hagan-Burke, \& Zou, 2009; Fletcher, Lyon, Fuchs, \& Barnes, 2007; Morais, 1997; National Reading Panel [NRP], 2000; Scheltinga, van der Leij, \& Struiksma, 2010). Contudo, coloca-se aos investigadores o desafio de perceber o tipo específico de relações entre estas variáveis e de saber se algumas delas se constituem como preditoras das outras (o que poderá ser muito relevante para o ensino da leitura ou para a prevenção e intervenção nas dificuldades da leitura; Denton et al., 2011; Kendeou, van den Broek, White, \& Lynch, 2009; Sindelar, Lane, Pullen, \& Hudson, 2002; Stanovich \& Cunningham, 1984).

As competências de leitura de palavras isoladas têm um papel fundamental na evolução da proficiência leitora, constituindo-se mesmo no elemento mais importante da competência leitora ${ }^{1}$ nos primeiros quatro anos de escolaridade (Jenkins, Fuchs, \& van den Broek, 2003; Juel, 1988). Em conjunto com a compreensão da fala, explicam praticamente toda a variância mensurável na competência leitora, explicando ainda uma percentagem muito significativa das dificuldades de leitura nessas idades (McBride-Chang, Manis, Seidenberg, Custodio, \& Doi, 1993; Simos, Breier, Fletcher, Bergman, \& Papanicolaou, 2000; Snow, Burns, \& Griffin, 1998). Uma das razões mais frequentemente apontadas para esta relação prende-se com o facto de a leitura eficaz de palavras isoladas libertar recursos atencionais que permitem à memória operatória incorporar elementos contextuais como a estrutura sintáctica, o desenvolvimento do tema ou outros elementos estruturais, sem ter que se ocupar com onerosos processos infra-lexicais (Perfetti, 1994; Perfetti, Rieben, \& Fayol, 1997).

A percepção da centralidade do reconhecimento de palavras na leitura levou os investigadores a dedicarem-se ao desenvolvimento de medidas deste constructo, no sentido de explorar a relação entre a leitura de palavras no texto, a leitura de palavras isoladas, a fluência e a compreensão, entre outros aspectos (Foorman, Francis, Fletcher, Schatschneider, \& Mehta, 1998; Foorman, Francis, Shaywitz, Shaywitz, \& Fletcher, 1997; Torgesen, 1998; Torgesen, Rashotte, \& Alexander, 2001). Os estudos realizados neste âmbito incluem uma diversidade considerável de tarefas (e.g., em alguns estudos recorre-se à leitura de listas de palavras isoladas que não estão incluídas em nenhum texto específico; noutros, as palavras a ler são retiradas de um texto específico, comparandose o desempenho da leitura das palavras no texto com a leitura das palavras isoladas) o que pode explicar, a par das variações na medição da velocidade da leitura, algumas das dificuldades encontradas na interpretação dos resultados (Jenkins et al., 2003).

\footnotetext{
${ }^{1}$ A competência leitora inclui os aspectos de descodificação, fluência e compreensão, de acordo com o nível de experiência do sujeito.
} 


\section{Reconhecimento de palavras isoladas (descontextualizadas) e reconhecimento de palavras no texto (contextualizadas)}

A fluência leitora, conceito que compreende (a) a precisão na descodificação das palavras, (b) o processamento automático e (c) a prosódia (Rasinski, 2003), e que é frequentemente avaliada como uma combinação de ritmo e de precisão de leitura ${ }^{2}$, depende em parte do reconhecimento individual (independente do contexto) de palavras mas também de elementos do contexto (Baker, 2000). Posner e Snyder (1975) sugerem a existência de dois processos de expectativas que influenciam a leitura de palavras num texto: um processo automático que permite o reconhecimento rápido das palavras através de uma rede semântica que é activada perante o estímulo constituído pela visualização das palavras; e um processo lento e oneroso para o sistema atencional a partir do qual o sujeito procura apoio no contexto para o reconhecimento de palavras. Estes dois processos, segundo Posner e Snyder (1975), permitem que o reconhecimento de palavras em contexto seja mais rápido e sustentado que o reconhecimento de palavras isoladas (situação em que as expectativas do que se seguirá são praticamente inexistentes).

O efeito facilitador do contexto no reconhecimento de palavras tem sofrido interpretações diversas porque enquanto alguns autores (e.g., Goodman , 1976; Smith, 1975, 1979) sustentam que os melhores leitores utilizam mais o contexto que os leitores fracos no reconhecimento de palavras, outros, baseados em estudos sobre tempos de reacção, defendem que são precisamente os leitores mais fracos quem mais recorre ao contexto para sustentar o acto de leitura (e.g., Perfetti, Goldman, \& Hogaboam, 1979; Perfetti, 1985; Swanson \& Alexander, 1997). Por outro lado, trabalhos em que as medidas utilizadas apresentam um carácter mais naturalista (i.e., em que se pede aos sujeitos que leiam um texto, eventualmente longo) têm chegado a resultados mistos: por um lado, sustentam a existência de efeitos do contexto, mas nem sempre verificam que são os leitores fracos os que mais recorrem ao contexto (Jenkins et al., 2003).

De acordo com Whitehurst e Lonigan (2001), o reconhecimento de palavras é activado a partir de processos denominados "outside in" e "inside out". Os primeiros utilizam informação exterior às palavras impressas para processar o seu significado (e.g., vocabulário, conhecimento de conceitos) e os segundos utilizam informação derivada das palavras impressas (e.g., conhecimento das letras, regras de correspondência grafema-fonema). Estes processos não se excluem mutuamente; são antes mutuamente suportativos (Whitehurst \& Lonigan, 2001). Conceptualmente, a sua formulação é semelhante à dos processos ascendentes ("bottom-up"; relativos ao impresso) e descendentes ("top-down"; relativos ao significado) enunciados no quadro do modelo interactivocompensatório desenvolvido por Stanovich (1996) para explicar as diferenças individuais na fluência leitora.

\footnotetext{
${ }^{2}$ A fluência é distinta da velocidade de leitura, constituindo esta última um aspecto específico da fluência. Velocidade de leitura e fluência na leitura são, pois, conceitos apenas parcialmente sobreponíveis. Diversos autores chamam, aliás, a atenção para o facto de provas como o Dynamic Indicators of Basic Early Literacy Skills (DIBELS; Good \& Kaminsky, 2002) serem frequentemente utilizados como testes de fluência quando medem apenas a componente de velocidade da fluência (Allington, 2006; Pressley, Hilden, \& Shankland, 2005; Samuels, 2006). Outros autores (e.g., Hudson, Pullen, Lane \& Torgesen, 2009) consideram que a variabilidade das definições de fluência não contribui para o esclarecimento terminológico na área e evidencia o quanto a fluência constitui um construto complexo e multifacetado.
} 
Contudo, no quadro deste último modelo, prevê-se explicitamente que, à medida que o leitor se torna mais eficiente, o reconhecimento de palavras se torna rápido e encapsulado, sendo muito pouco afectado por factores contextuais. Este facto poderá ser interpretado como um efeito de supremacia (em termos de velocidade) dos processos automáticos de activação semântica sobre os processos de predição consciente, o que resulta no reconhecimento das palavras por vias dos primeiros, antes que os segundos entrem em acção.

\section{Reconhecimento de tipos de palavras e fluência leitora}

Apesar de estar bem estabelecida a relação entre a leitura de palavras fora do contexto e no contexto (Shankweiler \& Liberman, 1972) e de se saber que o reconhecimento de palavras é o mais importante preditor da competência leitora (Jenkins et al., 2003), importa considerar eventuais relações diferenciais entre tipos de palavras e fluência leitora, em particular efeitos como a lexicalidade (que se refere ao facto de as palavras reais serem em geral mais rapidamente identificadas do que as pseudopalavras), a frequência (número médio de vezes que uma palavra ocorre numa determinada língua) ou a regularidade (palavras regulares são aquelas que podem ser lidas de acordo com as regras mais comuns de conversão grafo-fonémica; Salles \& Parente, 2002), uma vez que o acesso ao léxico mental depende não só de processos automáticos ou semiautomáticos de reconhecimento mas também de efeitos inerentes às características das próprias palavras (Ehri \& Wilce, 1983; Pollatsek , Juhasz, Reichle, Machacek, \& Rayner, 2008).

Efeitos como a lexicalidade, a frequência, a regularidade e até o comprimento, podem combinar-se de forma complexa na identificação de uma única palavra (Fernandes, Ventura, Querido e Morais, 2008; Lima e Castro, 2010). Por exemplo, a palavra "ginja" (2 sílabas, 5 letras), apesar de ser de pequeno comprimento, não é necessariamente lida com mais facilidade ou rapidez que a palavra "situação" (4 sílabas, 8 letras), devido a efeitos de valor de frequência (\# 5 para "ginja" e \# 5501 para "situação", de acordo com a CORLEX, - Léxico Multifuncional Computorizado do Português Contemporâneo) e de regularidade ("ginja" é uma palavra irregular", uma vez que o fonema / 3 / aparece duas vezes na palavra, sendo na primeiro traduzido pela letra "g" e na segunda pela mais provável letra “j”. "Situação”, por seu turno, é uma palavra regular, que apesar de longa, não levanta particulares problemas de leitura).

O efeito lexicalidade refere-se a uma diferença entre a leitura de palavras e pseudopalavras que prevê que as palavras sejam reconhecidas mais rapidamente do que as pseudopalavras ou não palavras, devido ao suporte semântico a elas associado (Ozubko, \& Joordens, 2011, Tellings, \& Bouts, 2011). Por outro lado a velocidade de leitura de pseudopalavras é apontada como um dos factores que mais claramente diferencia os maus leitores dos bons leitores, constituindo por isso um dos mais importantes preditores da competência leitora (Stanovich, 2000). Contudo, Thomson, Crewther e Crewther (2006) sugerem que nos quatro primeiros anos de escolaridade a leitura de pseudopalavras e de palavras representa o mesmo constructo (os autores encontraram correlações de 0.94 entre

\footnotetext{
${ }^{3}$ As palavras irregulares tendem a colocar maiores problemas ao leitor, uma vez que podem conter grafemas com valores fonémicos equívocos ou infrequentes. $\mathrm{Na}$ escrita, estas palavras levantam ainda maiores problemas.
} 
estes dois tipos de tarefas), diferenciando-se apenas posteriormente em função do aumento da fluência leitora (por via da automatização e do reconhecimento de palavras a partir do vocabulário e do conhecimento ortográfico e semântico; Thomson, Crewther, \& Crewther, 2006). Os resultados deste estudo merecem uma maior exploração, sobretudo numa perspectiva diferencial de línguas com estruturas e com níveis de consistência variados (e.g., de jong \& van der Leij, 2002; Landerl \& Wimmer, 2008; Lee, Tsai, Su, Tzeng, \& Hung, 2005; Reis, Faísca, Castro, \& Petersson, 2010; Simões, 2012).

Os efeitos de frequência e de regularidade das palavras têm sido estudados quer isoladamente, quer em termos de interacção. Genericamente os estudos têm evidenciado que a regularidade/consistência tem pouco impacto na velocidade e precisão de reconhecimento de palavras de elevada frequência, uma vez que estas podem ser recuperadas directamente do léxico mental antes de serem sujeitas a uma identificação através do (mais lento) sistema de conversão grafema-fonema (Coltheart, Curtis, Atkins, \& Haller, 1993; Coltheart, Rastle, Perry, Langdon, \& Ziegler, 2001; Lee, 2008). O mesmo não sucede, porém, na leitura de palavras de baixa frequência, situação em que se verifica um efeito de interação (Jared, 1997; Seidenberg, 1985; Seidenberg, Waters, Barnes, \& Tanenhaus, 1984; Sereno \& Rayner, 2000; Taraban \& McClelland, 1987; Waters \& Seidenberg, 1985). Inhoff e Topolsky (1994), por exemplo, num estudo sobre movimentos oculares, encontraram uma diferença de apenas 6 ms no reconhecimento de palavras regulares e irregulares de elevada frequência mas uma diferença de 17 ms no reconhecimento de palavras regulares e irregulares de baixa frequência.

Os estudos sobre o efeito do comprimento no reconhecimento de palavras têm encontrado resultados pouco consistentes, que no essencial variam entre o inibitório (as palavras mais extensas são mais difíceis de ler) e a ausência de efeito (e.g., Fernandes et. al., 2008; Lima \& Castro, 2010, no Português Europeu). New, Ferrand, Palier e Brysbaert (2006) realizaram uma revisão extensiva de trabalhos publicados entre 1951 e 2004 sobre decisão lexical, nomeação e movimentos oculares, em que foram utilizados como medidas os tempos de reacção, tendo encontrado sistematicamente resultados mistos. Outros estudos têm encontrado um efeito de comprimento (aumento do tempo de latência) na leitura de pseudopalavras mas não na leitura de palavras reais (e.g., Ans, Carbonnel, \& Valdois, 1998; Baciu et al., 2002; Ferrand, 2000; Weekes, 1997). Embora seja plausível supor que este efeito se deva à ausência de suporte lexical que, no caso das pseudopalavras, permita um processo rápido de identificação semântica, ainda não existe uma explicação cabal para a sua ocorrência (Juphard , Carbonnel, \& Valdois, 2004).

Os clássicos modelos de dupla via (Coltheart, Curtis, Atkins, \& 1993; Coltheart, Rastle, Perry, Langdon, \& Ziegler, 2001), por seu turno, prevêem efeitos diferenciais do comprimento para palavras e pseudopalavras na leitura de texto mas não em tarefas de decisão lexical (Coltheart et. al., 2001). Pelo contrário, os modelos PDP (Parallel Distributed Processing; Seidenberg, 1985; Seidenberg, Waters, Barnes, \& Tanenhaus, 1984) não prevêem qualquer efeito de comprimento na leitura de palavras e pseudopalavras, seja em contexto de leitura, seja em tarefas de decisão lexical, uma vez que defendem que todos os tipos de itens são processados globalmente, sem envolvimento de qualquer processo serial (Juphard, Carbonnel, \& Valdois, 2004). 
O presente estudo tem como objectivo fundamental estudar a relação entre o reconhecimento de tipos específicos de palavras (palavras frequentes, palavras raras, pseudopalavras, palavras regulares e palavras irregulares, organizadas por critérios de comprimento) e a velocidade leitora em três momentos distintos no tempo (final do $1 .{ }^{\circ}$ ano de escolaridade, meio do $2 .^{\circ}$ ano e final do $2 .^{\circ}$ ano). Complementarmente, são avaliadas as mudanças na velocidade leitora ao longo do tempo, é estimado em que medida os ganhos na velocidade de leitura são condicionados pelos valores iniciais de leitura e é analisada a mudança dos sujeitos no interior do grupo.

Duas hipóteses subjazem a este estudo: (a) a hipótese de a velocidade de leitura de texto poder ser diferencialmente explicada ou prevista pela capacidade de reconhecimento de palavras de tipos diversos (e.g. palavras raras, palavras irregulares) e (b) a hipótese de, num período de um ano, o poder explicativo da capacidade de reconhecimento de palavras de tipos diversos (relativamente à velocidade de leitura) variar ao longo do tempo (entre o final do $1^{\circ}$ e o final do $2^{\circ}$ ano de escolaridade). $\mathrm{O}$ esclarecimento destas questões poderá ser relevante, quer em termos da monitorização do desempenho dos alunos, quer em termos da estruturação do ensino geral da leitura, quer ainda em termos de intervenção junto de alunos com dificuldades nesta área. É ainda de realçar que estudos deste tipo, com línguas diferentes e com as variantes da mesma língua (como é o caso do Português Europeu e do Português do Brasil) não só não são redundantes como são indispensáveis, dadas as especificidades de consistência das relações grafo-fonémicas. Note-se a este propósito que alguns autores (e.g., Seymour et al., 2003) sustentam que o Português Europeu está mais próximo de ortografias europeias inconsistentes como o Inglês, o Francês ou o Dinamarquês, do que de ortografias relativamente transparentes como o Finlandês, o Italiano ou o Espanhol; outros (e.g., Sucena, Castro \& Seymour., 2009) acreditam que há um certo número de aspectos linguísticos que sugerem que o Português Europeu constitui uma ortografia de transparência intermédia.

\section{MÉTODO}

\section{Participantes}

No presente estudo participaram 95 alunos do $1 .^{\circ}$ ciclo, 48 do sexo masculino $(50,4 \%)$ e 47 do sexo feminino $(49,6 \%)$, de meio socioeconómico médio-alto, integrados em seis turmas, de três escolas, de três cidades distintas (Braga, Santo Tirso e Ponta Delgada). Os participantes são voluntários, constituindo pois uma amostra nãoprobabilística. Foram alvo do estudo todos os alunos que obtiveram autorização dos encarregados de educação para participar, tendo sido excluídos apenas os alunos referenciados para a educação especial. Duas das escolas são privadas e uma é do ensino público.

Por motivos diversos, houve oscilações significativas no número de alunos avaliados em cada um dos momentos. Especificamente, houve alunos que entraram de novo nas escolas após a primeira avaliação, outros que faltaram nos dias das provas e outros ainda que mudaram de turma ou de escola. Para além disso, houve alguns alunos que não realizaram a totalidade das provas previstas em algumas das administrações. Assim, no momento 1 (M1) foram avaliadas 105 alunos, no momento 2 (M2), 118 e no momento 3 (M3), 108. Os resultados referem-se apenas 
aos dos que realizaram as provas nos três momentos $(n=95)$, devendo no entanto salientar-se que em algumas provas específicas o número de alunos avaliados é inferior a 95.

\section{Medidas}

Foram utilizadas duas provas de leitura na avaliação das competências dos alunos: (1) prova de avaliação de reconhecimento de palavras escritas (TARPE; Moniz, 2008) e (2) prova de leitura de texto (em voz alta; REI; Carvalho, 2010).

(1) O TARPE é uma prova de reconhecimento de palavras composto por 100 itens, organizados em cinco subtestes, integrando palavras com diferentes atributos linguísticos: 20 palavras frequentes (com um total de 60 sílabas), 20 palavras raras (60 sílabas), 20 pseudopalavras (57 sílabas), 20 palavras regulares (61 sílabas) e 20 palavras irregulares (60 sílabas). Em cada grupo de tipologias de palavras, 10 são curtas e 10 são longas, obedecendo ao critério de comprimento. Os subgrupos de palavras do TARPE apresentam índices de consistência interna que variam entre os .82 para as pseudopalavras e os .90 para as palavras frequentes, com .83 para as palavras raras, .88 para as palavras regulares, .89 para as palavras irregulares. Moniz (2008) encontrou as seguintes correlações entre os subtestes do TARPE e a realização académica em Língua Portuguesa (classificação atribuída pelos professores) em alunos do $1 .{ }^{\circ}$ Ciclo do Ensino Básico: .49 para as palavras frequentes, .52 para as pseudopalavras, .56 para as palavras raras e para as palavras regulares e .57 para as palavras irregulares. Foi ainda encontrada uma correlação de .85 com a Prova de Leitura Rápida (Rebelo, 1993).

(2) Os alunos realizaram também a leitura em voz alta do texto "O REI" (forma A; Carvalho, 2010). Trata-se de um texto com 281 palavras para o qual existe um limite temporal de três minutos e do qual se extrai um índice de velocidade (número de palavras correctamente lidas por minuto) e de precisão (número de palavras correctamente lidas/número total de palavras lidas; este último indicador não foi considerado para efeitos deste estudo). Quando o sujeito lê o teste em menos de três minutos, o índice de velocidade leitora é também o número de palavras lidas por minuto. A fiabilidade da prova pelo método teste-reteste é de .94 .

\section{Procedimento}

Antes da aplicação das provas, quer as escolas, quer os professores, quer os encarregados de educação dos alunos foram contactos individualmente no sentido de autorizarem a administração das provas. Todos os encarregados de educação assinaram uma declaração de colaboração, tendo-se registado apenas cinco recusas.

As provas foram aplicadas individualmente, com intervalos aproximados de seis meses. A primeira aplicação foi realizada quando os alunos estavam no final do $1 .^{\circ}$ ano de escolaridade, a segunda no final do primeiro trimestre do $2 .^{\circ}$ ano de escolaridade e a terceira no final do $2 .^{\circ}$ ano de escolaridade. 
$\mathrm{Na}$ aplicação do TARPE, todas as palavras (itens) foram apresentadas de forma isolada, em formato electrónico (PowerPoint), não se tendo determinado tempo limite para a realização do mesmo. Porém, como a velocidade do reconhecimento de palavras constituiu uma dimensão na avaliação do desempenho, os sujeitos foram informados de que a sua velocidade de leitura seria alvo de avaliação. Como instrução, era pedido aos alunos que lessem em voz alta as palavras à medida que estas iam aparecendo no ecrã. Uma vez lida uma palavra, o avaliador, através de um clique, fazia aparecer imediatamente no ecrã a palavra seguinte. Saliente-se que apesar de as palavras (itens) serem lidas sequencialmente, sem paragens entre tipologias de palavras, os resultados devem ser calculados por blocos de tipologias de palavras (o somatório destes blocos permite naturalmente obter o tempo total despendido).

Na prova de leitura de texto foi solicitado aos alunos para lerem o texto em voz alta, o melhor que soubessem e de uma forma natural. Seguidamente foi-lhes apresentado o texto impresso em formato A4, letra Calibri, tamanho 12.

Para a gravação da leitura dos participantes foi utilizado o Audacity, software de edição digital de áudio.

\section{RESULTADOS}

Na Figura 1 são apresentadas as médias relativas ao tempo de leitura de cada uma das tipologias de palavras do TARPE nos três momentos de avaliação, verificando-se uma diminuição muito significativa do tempo despendido de M1 para M2 e posteriormente apenas uma ligeira diminuição.

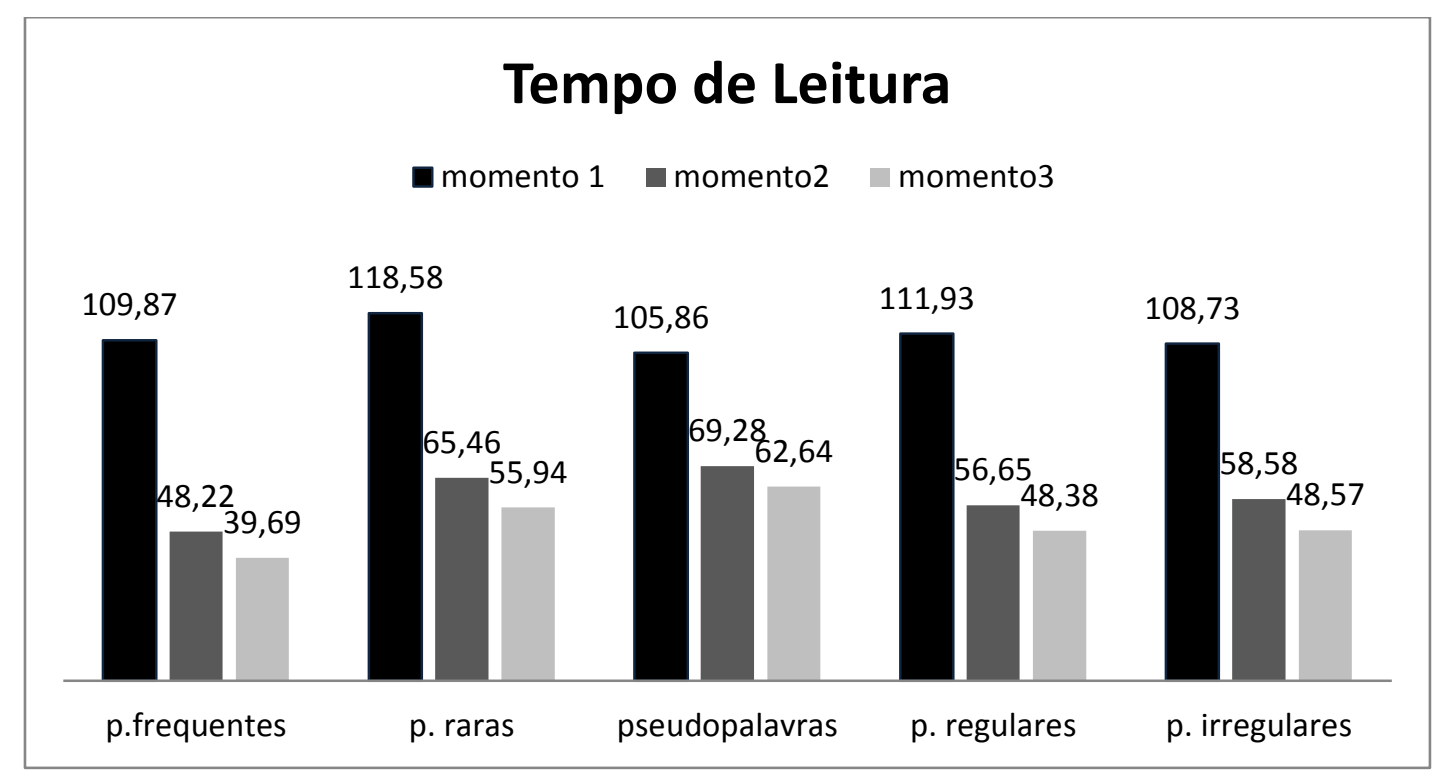

Figura 1. Média do Tempo de Leitura de Cada Tipologia de Palavras, em Segundos

Na Figura 2 apresenta-se a evolução da média do número de palavras lidas por minuto (leitura do texto REI). Tal como o gráfico sugere, a evolução da velocidade de leitura segue uma tendência linear de crescimento, $F(1,91)=$ $352.24, p<.01$. 


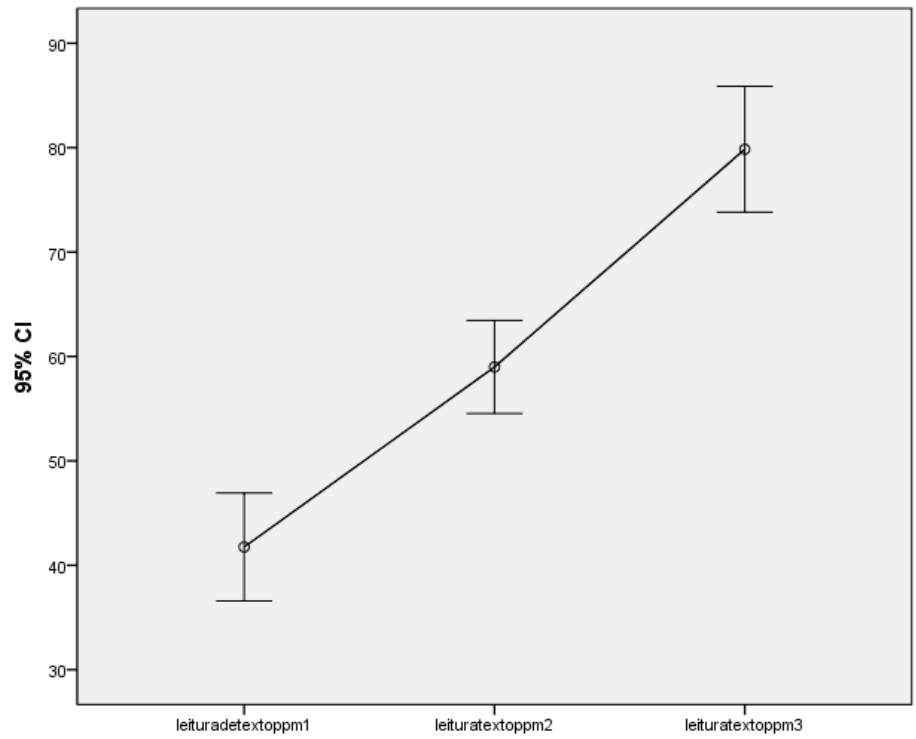

Figura 2. Média do Número de Palavras Lidas por Minuto ao Longo do Tempo (Leitura do Texto REI)

Nos quadros 1, 2 e 3, são apresentadas as correlações entre as variáveis independentes e variáveis dependentes nos três momentos de avaliação.

Quadro 1

Correlações de Pearson entre as Variáveis em M1

1. $2 . \quad 3 . \quad 4 . \quad 5$.

1. Palavras frequentes

2. Palavras raras $\quad .95^{* *}$

3. Pseudopalavras $\quad .86^{* *} \quad .92^{* *}$

4. Palavras regulares $.79^{* *} \quad .83^{* *} \quad .81^{* *}$

5. Palavras irregulares $.92^{* *} \quad .95^{* *} \quad .94^{* *} \quad .83^{* *}$

6. Leitura de texto $\quad-.65^{* *} \quad-.63^{* *} \quad-.62^{* *} \quad-.54^{* *} \quad-.64^{* *}$

Nota. ${ }^{* *} p<.01$

Quadro 2

Correlações de Pearson entre as Variáveis em M2

$\begin{array}{lllll}1 . & 2 . & 3 . & 4 . & 5 .\end{array}$

1. Palavras frequentes

2. Palavras raras $\quad .82^{* *}$

3. Pseudopalavras $\quad .80^{* *} \quad .92^{* *}$

4. Palavras regulares $.79^{* *} \quad .91^{* * *} \quad .88^{* * *}$

5. Palavras irregulares $.83^{* *} \quad .92^{* *} \quad .92^{* *} \quad .88^{* * *}$

6. Leitura de texto $\quad-.67^{* *} \quad-.77^{* *} \quad-.70^{* *} \quad-.72^{* *} \quad-.77^{* *}$

Nota. ${ }^{* *} p<.01$ 
Quadro 3

Correlações de Pearson entre as Variáveis em M3

1. 2.3 . 3 . 5 .

1. Palavras frequentes

2. Palavras raras $\quad .83^{* *}$

3. Pseudopalavras $\quad .78^{* *} \quad .90^{* *}$

4. Palavras regulares $.87^{* *} \quad .88^{* *} \quad .81^{* *}$

5. Palavras irregulares $.89^{* *} \quad .90^{* *} \quad .88^{* *} \quad .93^{* *}$

6. Leitura de texto $\quad-.59^{* *}-.66^{* *}-.69^{* *}-.63^{* *} \quad-.69^{* *}$

Nota. ${ }^{* * *} p<.01$

\section{Leitura isolada de palavras e velocidade leitora nos três momentos de avaliação}

Foram de seguida conduzidas diversas análises de regressão múltipla, com o intuito de explorar o valor preditivo da leitura de palavras isoladas relativamente à velocidade leitora nos diversos momentos de avaliação. Como se poderia supor a partir das elevadas correlações entre as variáveis preditoras (tipos de palavras), uma análise preliminar permitiu verificar um significativo efeito de colinearidade entre estas variáveis, tendo-se por isso efetuado regressões stepwise.

Foram realizadas três análises de regressão separadas para cada um dos momentos de avaliação, sendo a leitura de texto em cada um desses momentos a variável dependente e os tipos de palavras lidas em cada momento as variáveis preditoras. Em M1, reteve-se no modelo apenas a leitura de palavras frequentes, a qual explica $42 \%$ da variância na velocidade leitora, $\Delta R^{2}=.41, F(1,94)=58.22, p<.001$, com uma ponderação significativa de beta $(\beta=-.65, t=-7.63, p<.001)$. Saliente-se que neste primeiro momento, depois de controlado o efeito "turma frequentada pelos alunos", as tipologias de palavras reduzem o erro da predição em 32\%. Em M2, foram retidas no modelo as palavras raras, as quais explicam $42 \%$ da variância da velocidade leitora, $\Delta R^{2}=.41, F(1,95)=82.34, p$ $<.001(\beta=-.648, t=-9.07, p<.001)$. No segundo momento, depois de controlado o efeito "turma frequentada pelos alunos", as tipologias de palavras reduzem o erro da predição em 59\%. Por fim, em M3, o modelo explica $51 \%$ da variância da velocidade de leitura de texto, $\Delta R^{2}=.50, F(2,95)=50.17, p<.001$, sendo significativas as ponderações de beta para as pseudopalavras e para as palavras irregulares: $\beta=-.39, t=-2.63, p<.001$ para as pseudopalavras e $\beta=-.35, t=-2.33, p<.001$ para as palavras irregulares. No terceiro momento, depois de controlado o efeito "turma frequentada pelos alunos", as tipologias de palavras reduzem o erro da predição em $43 \%$.

\section{Leitura isolada de palavras no Momento 1 e velocidade leitora nos momentos seguintes de avaliação}

Foi igualmente explorada a relação entre a velocidade de leitura de palavras no momento 1 e a velocidade de leitura de texto nos momentos 1,2 e 3, no sentido de perceber se a relação se altera e, em caso afirmativo, em que sentido. O Quadro 4 mostra que as correlações entre a velocidade de leitura dos diversos tipos de palavras no 
primeiro momento e a velocidade de leitura de texto nos diversos momentos diminuem com o tempo, mas que apesar disso se mantêm significativas mesmo no terceiro momento. Considerando as diferenças entre correlações nos diversos momentos (e.g., diferença entre -.70 e -.47 nas palavras raras entre o momento 1 e o momento 3, que é a maior das diferenças entre correlações) nenhuma se revela significativa. Para cálculo do nível de significância destas diferenças foi utilizada a estatística $t$ de Chen e Popovich (2002), que permite a comparação de correlações relacionadas com a mesma entidade ou grupo em medidas repetidas no tempo.

Quadro 4

Correlaçães de Pearson entre Velocidade de Reconhecimento de Palavras no Momento 1 e Velocidade de Leitura nos Momentos Seguintes

P. frequentes $\quad$ P. raras $\quad$ Pseudopalavras $\quad$ P. regulares $\quad$ P. irregulares

\begin{tabular}{llllll}
\hline Leitura de texto M1 & $-.69^{* *}$ & $-.70^{* *}$ & $-.66^{* *}$ & $-.65^{* *}$ & $-.70^{* *}$ \\
Leitura de texto M2 & $-.52^{* *}$ & $-.48^{* *}$ & $-.52^{* *}$ & $-.64^{* *}$ & $-.52^{* *}$ \\
Leitura de texto M3 & $-.500^{* *}$ & $-.47^{* *}$ & $-.48^{* *}$ & $-.44^{* *}$ & $-.50^{* *}$ \\
\hline
\end{tabular}

Nota. ${ }^{* *} p<.01 ; \mathrm{M} 1$ - Primeiro momento; M2 - Segundo momento2; M3 - Terceiro Momento

\section{Estabilidade e mudança na velocidade leitora ao longo do tempo}

Para além da relação entre variáveis ao longo do tempo, pretendeu-se neste estudo testar formalmente o comportamento do conjunto dos participantes ao longo do tempo, no que diz respeito à velocidade leitora, bem como a estabilidade e mudança individual.

Do ponto de vista do conjunto dos sujeitos, as variações da velocidade leitora foram testadas através de uma ANOVA para medidas repetidas. O teste de Mauchly revelou que o pressuposto da esfericidade não está cumprido, $\chi^{2}(2)=11.63, p<.05$, pelo que os graus de liberdade foram corrigidos através da estimativa de esfericidade de Greenhouse-Geisser $(E=.89)$. Também os resultados dos testes multivariados, que não precisam de assumir a esfericidade para a obtenção de resultados correctos, evidenciam um efeito significativo do factor tempo (ou seja, do ensino) na velocidade leitora, $\Lambda$ de Wilks $=.201, F(1,91)=178.48, p<.001$ ). Os testes posthoc revelam que existem diferenças significativas na velocidade leitora entre os diferentes momentos de avaliação, sendo os ganhos entre M2 e M3 superiores $(F(1,91)=184.74, p<.01, r=.82)$ aos verificados entre M1 e M2 $(F(1,91)=108.15, p<.05, r=.74)$. Por outro lado, verifica-se que, em função do tempo, o comportamento dos valores da velocidade leitora é de natureza linear, $F(1,91)=352,24, p<.01$.

Do ponto de vista individual, a análise da estabilidade ou tracking das mudanças intraindividuais nas diferenças que ocorrem ao logo do tempo tem em consideração que os alunos não apresentam os mesmos valores de partida em M1, uma vez que o desvio-padrão nesse momento é de 24.66. É por isso previsível que a sua resposta ao ensino, ao longo do tempo de duração do estudo, seja diferenciada.

Na Figura 3 são apresentados em diagrama os resultados de velocidade leitora obtidos por 30 alunos (a apresentação de todos os alunos tornaria o diagrama incompreensível) em cada um dos momentos de avaliação. 
Foram escolhidos os dez alunos que obtiveram resultados mais baixos em M1 (à esquerda no diagrama), os dez que obtiveram resultados mais elevados (à direita no diagrama) e dez alunos com resultados médios em M1. Tomando apenas este grupo em consideração, é possível constatar, para além da heterogeneidade dos valores de partida, que nem sempre os valores iniciais condicionam as diferenças nos ganhos e que há sujeitos que no segundo momento lêem mais lentamente que no primeiro momento (isto apesar de a média dos alunos aumentar significativamente a velocidade do primeiro para o segundo momento), acelerando posteriormente.

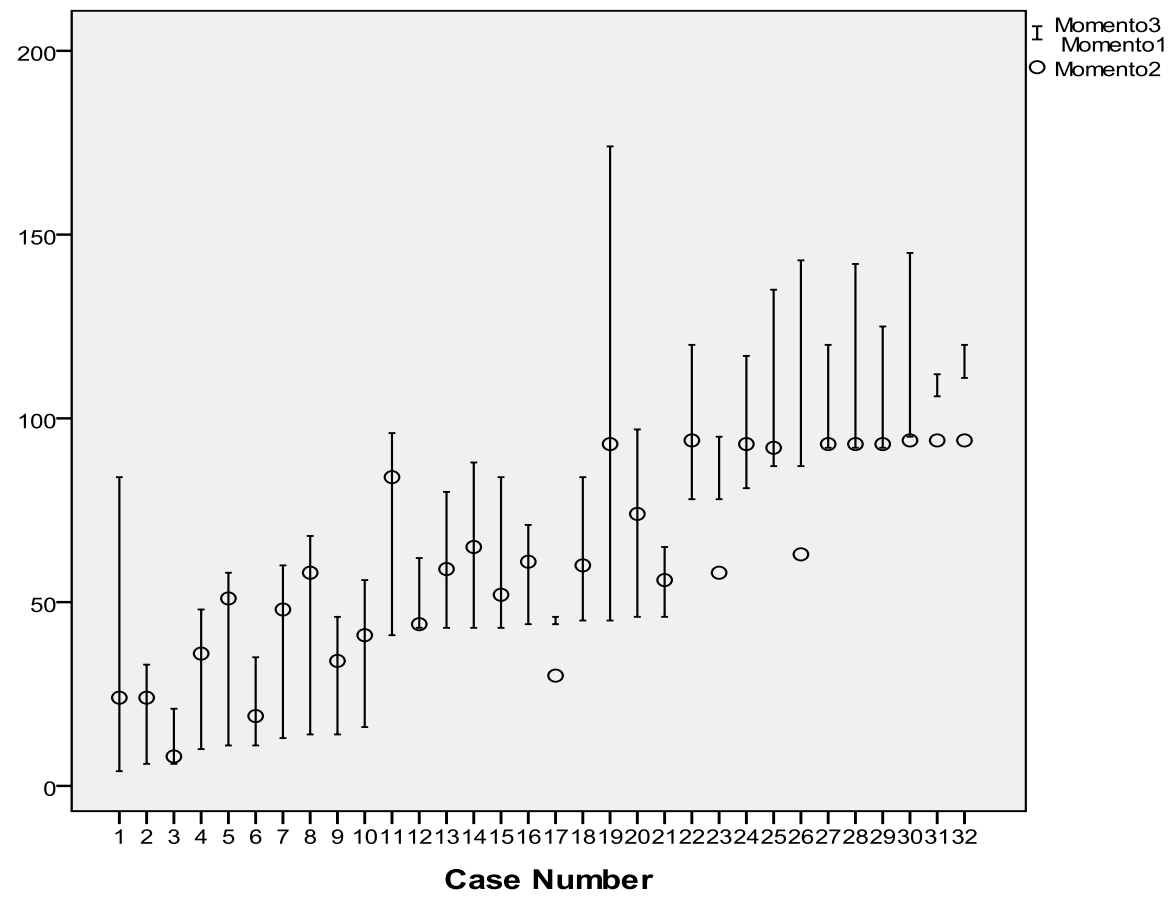

Figura 3. Diagrama de High-Low-Close

A análise da matriz de covariância mostra que a variância diminui de M1 para M2 (de 622.74 para 463. 66) mas aumenta significativamente de M2 para M3 (de 463.66 para 847.01). A Figura 4 permite perceber precisamente uma diminuição da heterogeneidade dos resultados de M1 para M2, seguida de um aumento M2 para M3. Por outro lado, é possível verificar que de M1 para M3 há cruzamentos nas trajetórias interindividuais, o que indica que nem sempre os valores iniciais condicionam os valores finais. Contudo, os valores das correlações entre M1 e M2 $(r=.78)$ e entre M2 e M3 $(r=.87)$ evidenciam a existência de uma estabilidade moderada a elevada das mudanças intraindividuais nas diferenças interindividuais entre cada momento (bem acima do valor requerido para um traço ou característica evidenciar tracking: $r \geq .50)$ e o coeficiente de correlação intraclasse $=.91,($ IC95\% $=$ $.88 ; .94)$ sugere uma estabilidade global moderada a elevada da posição de cada participante no grupo.

Os tipos de palavras que nos diversos momentos se revelaram preditores significativos da velocidade leitora também apresentam variações significativas ao longo do tempo para o conjunto dos sujeitos mas, ao contrário do que sucede com a leitura de texto, as variâncias diminuem em todos os momentos de avaliação para qualquer destes preditores. Assim, para as palavras frequentes: $\Lambda$ de Wilks $=.53, F(1,67)=29.50, p<.001$, com diferenças significativas entre M1/M2, $F(1,67)=36.72, p<.01, r=.35$ e entre $\mathrm{M} 2 / \mathrm{M} 3, F(1,67)=32.71, p<.01, r=.32$; para as palavras raras, $\Lambda$ de Wilks $=.54, F(1,67)=28.29, p<.001$, com diferenças significativas entre M1/M2, $F$ 
$(1,67)=40.52, p<.01, r=.37$ e entre M2/M3, $F(1,67)=23.64, p<.01, r=.23$; para as palavras irregulares, $\Lambda$ de Wilks $=.47, F(1,65)=36.49, p<.001$, com diferenças significativas entre M1/M2, $F(1,65)=52.63, p<.01, r$ $=.44$ e entre M2/M3, $F(1,65)=34.55, p<.01, r=.35$; para as pseudopalavras, $\Lambda$ de Wilks $=.56, F(1,67)=$ 25.98, $p<.001$, com diferenças significativas entre M1/M2, $F(1,67)=52.63, p<.01, r=.44$ e entre M2/M3, $F$ $(1,67)=34.55, p<.01, r=.35$.
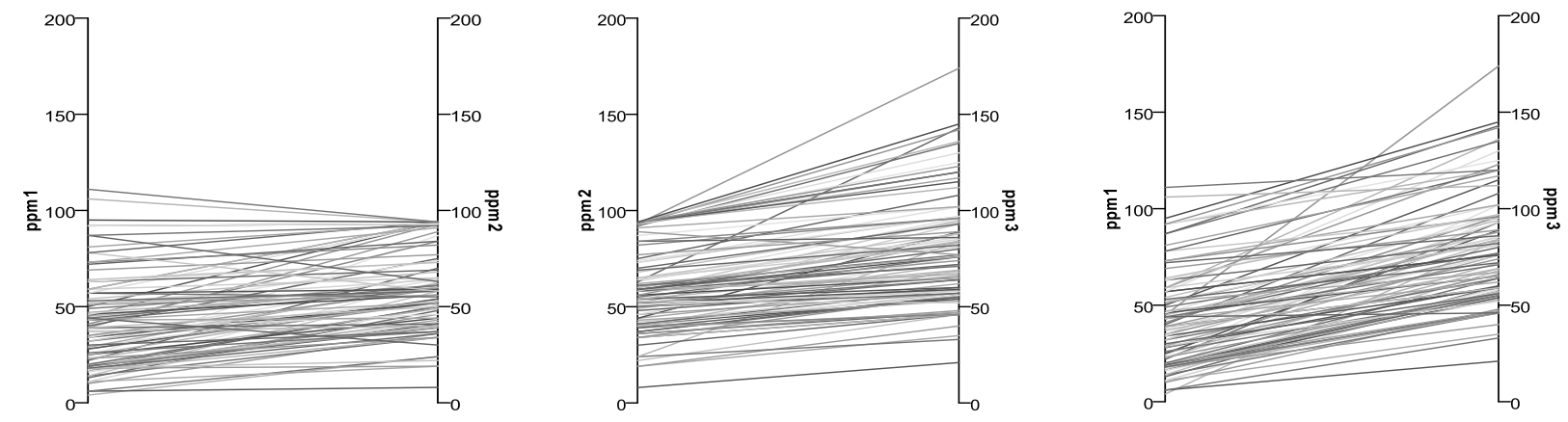

Figura 4. Trajetórias Interindividuais em Velocidade de Leitura de Texto

Do ponto de vista das trajectórias individuais, as matrizes de covariância evidenciam um comportamento algo diferente do que se verifica com a leitura de texto, já que a variância diminui de forma significativa entre todos os momentos, ainda que a diminuição de M1 para M2 seja muito superior (palavras frequentes: 9723.38 em M1, 636.23 em M2 e 355.15 em M3; palavras raras: 7879.02 em M1, 760.73 em M2 e 664.84 em M3; palavras irregulares: 5904.58 em M1, 759.07 em M2 e 585.51 em M3; pseudopalavras: 4276.71 em M1, 789.82 em M2 e 485.32 em M3; cf. Figura 5).
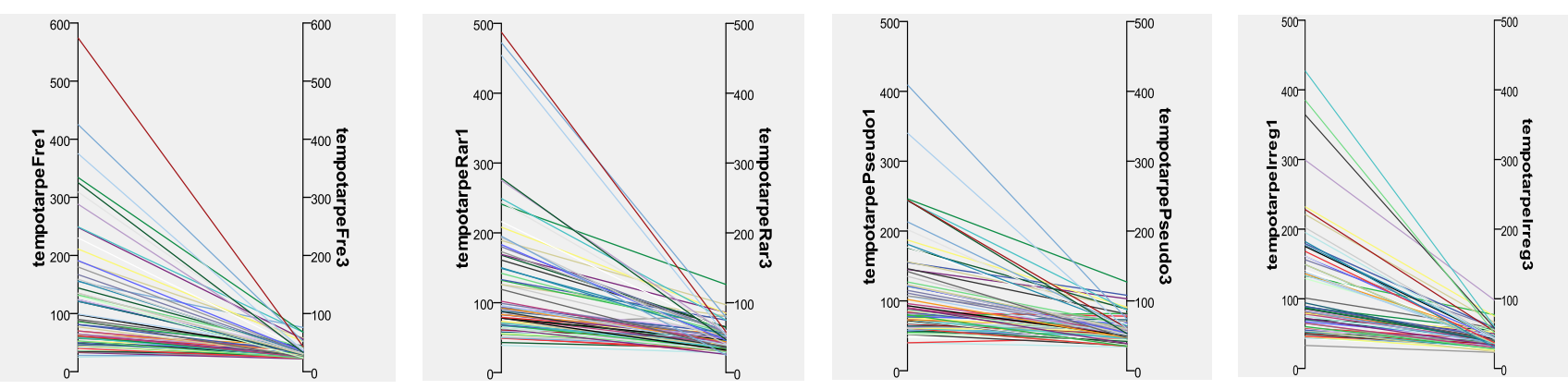

Figura 5. Trajetórias Interindividuais em Leitura de Palavras Isoladas (Palavras Frequentes, Palavras Raras, Pseudopalavras, Palavras Irregulares)

\section{DISCUSSÃO}

\section{Reconhecimento de palavras isoladas e velocidade de leitura de texto}

Os nossos resultados não diferem dos resultados encontrados em investigações anteriores no que diz respeito à relação entre leitura de palavras isoladas e velocidade de leitura de texto (Burke et. al, 2009; Cochrane, 1974; Coltheart et. al. 2001; Biemiller, 1977-78; Jenkins et. al. 2003; Stanovich, 1996, 2000). Utilizando como 
referência o clássico modelo de Posner e Snyder (1975), podemos considerar que os resultados indicam que mais de metade da variância da velocidade leitora é explicada pela capacidade de leitura descontextualizada de palavras (um processo de reconhecimento rápido assente na activação uma rede semântica de representações) podendo o restante ser explicado, pelo mesmo parcialmente, por factores contextuais da leitura de texto.

Os resultados mostram ainda que os eventuais efeitos de lexicalidade, frequência, regularidade e comprimento do reconhecimento de palavras isoladas na velocidade de leitura de texto existem, mas são equívocos. Considerado isoladamente, qualquer dos tipos de palavras prediz significativamente a velocidade de leitura de texto. Contudo, quando inscritos num modelo de regressão múltipla, nenhum dos tipos de palavras aduz poder explicativo à primeira variável inscrita no modelo. Assim, em M1 as palavras frequentes emergem como o melhor preditor da velocidade de leitura, não aduzindo os restantes tipos de palavras significância específica ao modelo. $\mathrm{O}$ mesmo sucede em M2 com as palavras raras e em M3 com as pseudopalavras e as palavras irregulares. A flutuação do melhor preditor em cada momento de avaliação é aparentemente aleatória, ainda que possa ser explicada pelas elevadas correlações entre os diferentes tipos de palavras nos diversos momentos, as quais apontam para um importante efeito de colinearidade entre estas variáveis. Ou seja, parece haver um importante efeito preditor da leitura de palavras mas não necessariamente de um tipo específico de palavras.

No que diz respeito a eventuais efeitos de lexicalidade, um dos mais frequentemente referidos na literatura (e.g., Beech \& Awaida, 1992; Coltheart et al. 2001; Simos, Breier, Fletcher, Foorman, Castillo, \& Papanicolaou, 2002), deve acentuar-se que a leitura de pseudopalavras apresenta um poder preditivo da velocidade de leitura idêntico ao dos outros tipos de palavras em todos os momentos. A este propósito, Thomson, Crewther, e Crewther (2006), encontraram correlações de .94 entre leitura de pseudopalavras e leitura de outros tipos de palavras nos quatro primeiros anos de escolaridade, o que significa, segundos os autores, que a leitura de pseudopalavras não representa um constructo suficientemente específico, ao contrário do que é vulgarmente referido na literatura. Daí que afirmem também que os resultados do seu estudo demonstram que as tarefas de leitura de pseudopalavras não fornecem informação mais útil do que as tarefas de leitura de palavras no diagnóstico ou no prognóstico de leitura de texto. Estudos como o de Bosse e Valdois (2003) e o de Zabell e Everatt (2002) (este último realizado com adultos) suportam esta posição.

Do mesmo modo, não encontramos um efeito claro da regularidade/irregularidade de leitura de palavras, o que está em linha com o que tem sido referido noutros trabalhos (e.g., Coltheart, Curtis, Atkins, \& Halller, 1993; Coltheart et al., 2001; Lee, 2008). Para além disso e contrariamente ao que se tem verificado em estudos nesta área (e.g., Jared, 1997; Seidenberg, 1985; Seidenberg, et al. 1984; Sereno \& Rayner, 2000; Taraban \& McClelland, 1987; Waters \& Seidenberg, 1985), este efeito não se verifica sequer na leitura de palavras raras. É possível que muitos sujeitos utilizem uma estratégia de leitura silábica que funciona relativamente bem na leitura de listas de palavras isoladas (seja qual for o tipo) mas se revela menos funcional na leitura de texto, onde as estratégias antecipatórias e a sobrecarga continuada do sistema atencional são muito mais significativas. No quadro do modelo de memória multitraço de Ans, Carbonel e Valdois (1998), seria possível afirmar que na leitura de palavras isoladas é sustentável o recurso ao sistema analítico de leitura (o qual, teoricamente, é activado quando o 
sistema global de leitura, que abarca a totalidade da palavra, falha, ou quando não é activável porque o leitor é inexperiente), independentemente da sua forma, o mesmo não sucedendo na leitura de texto, que exige um processo global e sustentado de abordagem da tarefa. Isto é tanto mais plausível quanto no Português Europeu a sílaba constitui uma poderosa e pregante unidade infralexical de suporte da leitura de palavras isoladas, independentemente da compreensão do seu significado (Sucena \& Castro, 2008).

Apesar de tudo, o facto de as palavras irregulares predizerem a velocidade de leitura de texto no terceiro momento, pode indiciar que o acesso ao léxico poderá estar já a ser realizado por uma via lexical e não por uma via infralexical. Um eventual prolongamento da recolha de dados no tempo poderia ter esclarecido esta possibilidade.

Também não foi encontrado nenhum efeito específico do comprimento das palavras isoladas na leitura de texto. Relembre-se, a este propósito, que a extensiva revisão de literatura realizada por New et al. (2006), relativa a estudos publicados entre 1951 e 2004, encontrou sistematicamente resultados mistos quanto a este efeito, pelo que os nossos resultados poderão sustentar a tendência da ausência de efeito.

É ainda particularmente relevante notar o comportamento da variância dos resultados no que diz respeito à leitura de palavras isoladas e à leitura de texto. Assim, enquanto na leitura de texto há uma diminuição da variância de M1 para M2, seguida de um aumento para M3 (ver Figura 4), na leitura de palavras isoladas há um decréscimo muito significativo da variância de M1 para M2, seguido de novo, mas muito mais moderado, decréscimo de M2 para M3 (Figura 5). Se considerarmos apenas o comportamento da variância de M1 para M3, constataremos que enquanto a variância dos resultados na leitura de texto aumenta, na leitura de palavras de palavras diminui (fundamentalmente pelos progressos muito significativos dos sujeitos que em M1 obtiveram resultados piores). Este facto fica provavelmente a dever-se à impossibilidade de os sujeitos, no contexto de leitura de palavras isoladas, mobilizarem o processo automático de activação semântica em rede, o qual diminui a necessidade de mobilização de recursos atencionais para a tarefa, bem como o patamar de percepção das palavras, com consequente aumento da velocidade de leitura (Jenkins et. al., 2003; Posner \& Snyder, 1975).

\section{Estabilidade e mudança na velocidade leitora ao longo do tempo}

A análise da estabilidade ou tracking aponta para uma estabilidade moderada a elevada das mudanças intraindividuais nas diferenças interindividuais, o mesmo sucedendo quanto à da posição dos participantes em velocidade leitora nos diversos momentos. Apesar disso, é possível verificar a existência de trajectórias que se cruzam ao longo do tempo (Figura 5) o que significa que, se os valores iniciais condicionam em geral os resultados posteriores dos sujeitos, existem casos em que sujeitos com piores desempenhos à partida melhoram significativamente e outros em que sujeitos com valores iniciais mais elevados progridem mais lentamente do que seria de esperar. A Figura 3 é a este respeito elucidativa.

Estes resultados são no essencial idênticos aos encontrados em estudos clássicos como os de Juel (1988), ou em estudos mais recentes e em línguas com diferentes níveis de transparência como os de Landerl e Wimmer (2008), Bast e Reitsma (1998), Cossu, Giuliotta e Marshall (1995), entre outros. É pois possível supor que, pelo menos 
para um número significativo de sujeitos, o nível de competência leitora no final do primeiro ano é preditivo da competência posterior, o que pode significar que o ensino, ou outro qualquer factor, após um certo tempo, tem uma menor probabilidade de alterar a posição relativa dos sujeitos.

\section{Conclusão Geral}

No seu conjunto, os resultados deste estudo revelam a importância do reconhecimento de palavras isoladas na velocidade de leitura de texto mas evidenciam padrões opostos no comportamento da variância dos resultados ao longo do tempo. Enquanto no reconhecimento de palavras isoladas as diferenças entre os sujeitos tendem a diminuir drasticamente, na velocidade de leitura de texto tendem a aumentar. Por outro lado, as posições relativas dos sujeitos na velocidade leitora são tendencialmente estáveis, ainda que haja excepções. Estes são porventura os resultados mais relevantes desta investigação, sobretudo no que diz respeito ao Português Europeu, língua em que praticamente não existem estudos longitudinais com este desenho.

A diminuição das diferenças na leitura de palavras isoladas, acompanhada pelo aumento das diferenças na velocidade da leitura de texto, fazem supor que, no texto, a maior rapidez nas conexões interlexicais beneficiará quer do conhecimento sintáctico, quer do conhecimento semântico, quer ainda de dispositivos de interpretação sistemática do texto. É, pois, de supor que, embora a velocidade e a fluência da leitura assentem na identificação isolada de palavras, há outro aspectos que, com o tempo, contribuirão progressivamente para explicar o aumento das diferenças na velocidade de leitura de texto. A tendência para a estabilidade das posições relativas dos sujeitos, por seu turno, evidencia que a instrução não introduz variações significativas nas posições relativas dos alunos depois do $1 .^{\circ}$ ano de escolaridade.

Do ponto de vista do ensino da leitura, os resultados sugerem a importância do treino de leitura de palavras individuais, uma vez que uma palavra só pode ser reconhecida automaticamente se a sua ortografia, pronúncia e significado estiverem firmemente representadas e interligadas na memória. Todas são necessárias; nenhuma é suficiente (Anglin, 1993). Porém, e ainda mais importante, os resultados sugerem que, para além da importância do reconhecimento de palavras isoladas, o treino de texto conectado é indispensável para o desenvolvimento dos processos automáticos de activação semântica em rede. A conjugação do treino de leitura individual de palavras de um texto, com o treino de leitura dessas palavras conectadas em texto poderá otimizar o conhecimento lexical e, por consequência, a compreensão do texto.

Por fim, os resultados sugerem a importância do desenvolvimento de sistemas de apoio precoce a crianças que, no início da aprendizagem, apresentam algumas dificuldades. $\mathrm{O}$ facto de, em geral, os alunos se manterem nas suas posições relativas e de as diferenças de desempenho na velocidade de leitura de texto aumentarem ao longo do tempo mostra que, na ausência de uma intervenção atempada e deliberada, a situação tende a não se alterar. 


\section{Limitações do estudo}

Importa considerar, como limitação principal do estudo, que este inclui apenas três medidas repetidas no tempo. A continuação da recolha de dados, que está em curso, poderá revelar realidades diferentes das até aqui encontradas. Será por exemplo importante saber se os tipos de palavras que se relevaram os melhores preditores da velocidade leitora em M3 continuarão a ser os melhores preditores nos momentos de avaliação seguintes. Ou se um padrão relativamente aleatório relativamente aos melhores preditores acabará por consolidar a ideia de que o reconhecimento de palavras isoladas constitui um excelente preditor da velocidade leitora independentemente do tipo de palavras utilizadas como estímulo.

Saliente-se, por fim, as oscilações no número de provas realizadas nos três momentos, o que nos levou a eliminar os sujeitos que não realizaram todas as provas, com a consequente significativa redução do número final de participantes.

\section{REFERÊNCIAS}

Allington, R. L. (2006). What really matters for struggling readers: Designing research-based programs (2nd ed). Boston: Allyn \& Bacon.

Anglin, J. M. (1993). Vocabulary knowledge. In H. Singer \& R.B.Ruddell (Eds.), Theoretical Models and Process of Reading ( $3^{\text {rd }}$ ed., pp. 343-371). Newark, DE: IRA.

Ans, B., Carbonnel, S., \& Valdois, S. (1998). A connectionist multipletrace memory model for polysyllabic word reading. Psychological Review, 105, 678-723.

Baciu, M., Ans, B., Carbonnel, S., Valdois, S., Juphard, A., Pachot-Clouard, M., Segebarth, C. (2002). Length effect during word and pseudo-word reading: An event-related fMRI study. Neuroscience Research Communications, 30, 155165.

Baker, L. (2000). Building the word-level foundation for engaged reading. In L. Baker, M. J. Dreher \& J. T. Guthrie (Eds.), Engaging young readers: Promoting achievement and motivation (pp. 17-42). New York: Guilford Press.

Bast, J., \& Reitsma, P. (1998). Analyzing the development of individual differences in terms of Matthew Effects in reading: Results from a Dutch longitudinal study. Developmental Psychology, 34, 1373-1399.

Beech, J. R., \& Awaida, M. (1992). Lexical and nonlexical routes: A comparison between normally achieving and poor readers. Journal of Learning Disabilities, 25, 196-206.

Biemiller, A. (1977-78). Relationship between oral reading rates for letters, words, and simple text in the development of reading achievement. Reading Research Quarterly, 13, 223-253.

Bosse, M., \& Valdois, S. (2003). Patterns of developmental dyslexia according to a multi-trace memory model of reading. Current Psychology Letters, 10. Retirado de http://cpl.revues.org/document92.html

Burke, M. D., Crowder, W., Hagan-Burke, S., \& Zou, Y. (2009). A comparison of two path models for predicting reading fluency. Remedial and Special Education, 30(2), 84-95.

Carvalho, A. (2010). O REI - Teste de avaliação da fluência e precisão de leitura. Vila Nova de Gaia: Edipsico.

Chen, P. Y., \& Popovich, P. M. (2002). Correlation: Parametric and nonparametric measures. Newbury Park, CA: Sage Publications. 
Cochrane, R. G. (1974). The effect of context on word recognition. The Slow Learning Child, 21, 38-43.

Coltheart, M., Curtis, B., Atkins, P., \& Haller, M. (1993). Models of reading aloud: Dual-route and parallel-distributedprocessing approaches. Psychological Review, 100, 589-608.

Coltheart, M., Rastle, K., Perry, C., Langdon, R., \& Ziegler, J. (2001). DRC: A dual route cascaded model of visual word recognition and reading aloud. Psychological Review, 108, 204-256.

Cossu, G., Giuliotta, M., \& Marshall, J. C. (1995). Acquisition of reading and written spelling in a transparent orthography: Two non-parallel processes? Reading and Writing, 7, 9-22.

De Jong, F. H., \& Van der Leij, A. (2002). Effects of phonological abilities and linguistic comprehension on the development of reading. Scientific Studies of Reading, 6, 51-77.

Denton, C. A., Barth, A. E., Fletcher, J. M., Wexler, J., Vaughn, S., Cirino, P. T, . . Francis, D. J. (2011). The relations among oral and silent reading fluency and comprehension in middle school: Implications for identification and instruction of students with reading difficulties. Scientific Studies of Reading, 15, 109-135.

Ehri, L., \& Wilce, L. S. (1983). Development of word identification speed in skilled and less skilled beginning readers. Journal of Educational Psychology, 75, 3-18.

Fernandes, S., Ventura, P., Querido, L., \& Morais, J. (2008). Reading and spelling acquisition in European Portuguese: A preliminary study. Reading and Writing, 21, 805-821.

Ferrand, L. (2000). Reading aloud polysyllabic words and nonwords: The syllabic length effect reexamined. Psychonomic Bulletin and Review, 7, 142-148.

Fletcher, J. M., Lyon, G. R., Fuchs, L. S., \& Barnes, M. A. (2007). Learning disabilities: From identification to intervention. New York: Guilford Press.

Foorman, B. R., Francis, D. J., Fletcher, J. M., Schatschneider, C., \& Mehta, P. (1998). The role of instruction in learning to read: Preventing reading failure in at-risk children. Journal of Educational Psychology, 68, 70-74.

Foorman, B. R., Francis, D. J., Shaywitz, S. E., Shaywitz, B. A., \& Fletcher, J. M. (1997). The case for early reading intervention. In B. Blachman (Ed.), Foundations of reading acquisition and dyslexia (pp. 243-264). New Jersey: Erlbaum.

Good, R.H., \& Kaminsky, R. A. (2002). Dynamic indicators of basic literacy skills (6th ed.). Eugene, OR: Institute for the Development of Educational Achievement.

Goodman, K. S. (1976). A psycholinguistic guessing game. In H. Singer \& R. Ruddell (Eds.), Theoretical models and processes of reading (2nd ed., pp. 497-508). Newark, DE: International Reading Association.

Hudson, R. F., Pullen, P. C., Lane, H. B., \& Torgesen, J. K. (2009). The complex nature of reading fluency: A multidimensional view. Reading \& Writing Quarterly, 25, 4-32.

Inhoff, A. W., \& Topolski, R. (1994). Use of phonological codes during eye fixations in reading and in on-line and delayed naming tasks. Journal of Memory and Language, 33, 689-713.

Jared, D. (1997). Spelling-sound consistency affects the naming of high-frequency words. Journal of Memory and Language, 36, 505-529.

Jenkins, J. R., Fuchs, L. S., \& van den Broek, P. (2003). Sources of individual differences in reading comprehension and reading fluency. Journal of Educational Psychology, 13, 719-729.

Juel, C. (1988). Learning to read and write: A longitudinal study of fifty-four children from first through fourth grade. Journal of Educational Psychology, 80, 437-447.

Juphard, A., Carbonnel, S., \& Valdois, S. (2004). Length effect in reading and lexical decision: Evidence from skilled readers and a developmental dyslexic participant. Brain and Cognition, 55, 332-340. 
Kendeou, P., van den Broek, P., White, M. J., \& Lynch, J. S. (2009). Predicting reading comprehension in early elementary school: The independent contributions of oral language and decoding skills. Journal of Educational Psychology, $101,765-778$.

Landerl, K., \& Wimmer, H. (2008). Development of word reading fluency and spelling in a consistent orthography. Journal of Educational Psychology, 100, 150-161.

Lee, C.-Y. (2008). Rethinking of the regularity and consistency effects in reading. Language and Linguistics 9, 77-186.

Lee, C., Tsai, J., Su, E. C., Tzeng, O. J. L., \& Hung., D. L. (2005). Consistency, regularity, and frequency effects in naming Chinese characters. Language and Linguistics, 6, 75-107.

Lima, C. F., \& Castro, S. L. (2010). Reading strategies in orthographies of intermediate depth are flexible: Modulation of length effects in Portuguese. European Journal of Cognitive Psychology, 22, 190-215, doi:10.1080/09541440902750145

McBride-Chang, C., Manis, F. R., Seidenberg, M. S., Custodio, R. G., \& Doi, L. M. (1993). Print exposure as a predictor of word reading and reading comprehension in disabled and nondisabled readers. Journal of Educational Psychology, $85,230-238$.

Moniz, A. V. (2008). Reconhecimento de palavras escritas e competência leitora: Construção e validação do teste de avaliação do reconhecimento de palavras escritas (T.A.R.P.E.; Tese de Doutoramento não publicada). Universidade dos Açores: Ponta Delgada.

Morais, J. (1997). A arte de ler: Psicologia cognitiva da leitura. Lisboa: Edições Cosmos.

New, B., Ferrand, L., Palier, C., \& Brysbaert, M. (2006). Reexamining the word length effect in visual word recognition: New evidence from the English Lexicon Project. Psychonomic Bulletin \& Review, 13, 45-52.

National Reading Panel (2000). Teaching children to read: An evidence-based assessment of the scientific research literature on reading and its implications for reading instruction. Washington, DC: National Institute of Child Health and Human Development.

Ozubko, J. D., \& Joordens, S. (2011). The similarities (and familiarities) of pseudowords and extremely high-frequency words: Examining a familiarity-based explanation of the pseudoword effect. Journal of Experimental Psychology: Learning Memory and Cognition 37, 123-139.

Perfetti, C. A. (1985). Reading skill. Hillsdale: Lawrence Erlbaum.

Perfetti, C. A. (1994). Psycholinguistics and reading ability. In M. A. Gernsbacher (Ed.), Handbook of psycholinguistics (pp. 849-894). San Diego, CA, US: Academic Press.

Perfetti, C. A., Goldman, S. R., \& Hogaboam, T. W. (1979). Reading skill and the identification of words in discourse context. Memory \& Cognition, 7, 273-282.

Perfetti, C. A., Rieben, L., \& Fayol, M. (Eds.). (1997). Learning to spell: Research, theory, and practice across languages. Mahwah, NJ: Lawrence Erlbaum Associates.

Pollatsek, A., Juhasz, B. J., Reichle, E. D., Machacek, D., \& Rayner, K. (2008). Immediate and delayed effects of word frequency and word length on eye movements in reading: A reversed delayed effect of word length. Journal of Experimental Psychology: Human Perception and Performance, 34, 726-750.

Posner, M. I., \& Snyder, R. R. (1975). Attention and cognitive control. In R. L. Solso (Ed.), Information processing and cognition: The Loyola Symposium (pp. 55-85). Hillsdale, NJ: Erlbaum.

Pressley, M., Hilden, K., \& Shankland, R. (2005). An evaluation of end-of-grade 3 Dynamic Indicators of Basic Early Literacy Skills (DIBELS): Speed reading without comprehension, predicting little. East Lansing, MI: Michigan State University Literacy Achievement Research Center.

Rasinski, T. V. (2003). The fluent reader. New York: Scholastic. 
Rebelo, J. A. (1993). Dificuldades da leitura e da escrita em alunos do ensino básico (Vol. 5). Porto: ASA.

Reis, A., Faísca, L., Castro, S.-L., \& Petersson, K. M. (2010). Preditores da leitura ao longo da escolaridade: Um estudo com alunos do $1^{\text {o }}$ ciclo do ensino básico. In C. Nogueira, I. Silva, L. Lima, A. T. Almeida, R. Cabecinhas, R. Gomes, C. Machado, A. Maia, A. Sampaio, \& M. C. Taveira (Eds.), Actas do VII Simpósio Nacional de Investigação em Psicologia (pp. 3117-3132). Braga: Universidade do Minho.

Salles, J. F., \& Parente, M. A. M. P. (2002). Processos cognitivos na leitura de palavras em crianças: Relações com compreensão e tempo de leitura. Psicologia: Reflexão e Crítica, 15, 321-331.

Samuels, S. J. (2006). Toward a model ofreading fluency. In S. J. Samuels \& A. E. Farstrup (Eds.), What research has to say about fluency instruction (pp. 24- 46). Newark, DE: International Reading Association.

Scheltinga, F., van der Leij, A., \& Struiksma, C. (2010). Predictors of response to intervention of word reading fluency in Dutch. Journal of Learning Disabilities, 43, 212-228.

Seidenberg, M. S. (1985). The time course of phonological code activation in two writing systems. Cognition 19, 1-30.

Seidenberg, M. S., Waters, G. S., Barnes, M., \& Tanenhaus, M. (1984). When does irregular spelling or pronunciation influence word recognition? Journal of Verbal Learning and Verbal Behavior 23, 383-404.

Sereno, S. C., \& Rayner, K. (2000). Spelling-sound regularity effects on eye fixations in reading. Perception and Psychophysics, 62, 402-409.

Seymour, P. H. K., Aro, M., \& Erskine, J. M. (2003). Foundation literacy acquisition in European orthographies. British Journal of Psychology, 94, 143-174.

Shankweiler, D., \& Liberman, I. Y. (1972). Misreading: A search for causes. In J. Kavanagh \& I. Mattingly (Eds.), Language by ear and eye. Cambridge, MA: MIT Press.

Simos, P. G., Breier, J. I., Fletcher, J. M., Bergman, E., \& Papanicolaou, A. C. (2000). Cerebral mechanisms involved in word reading in dyslexic children: A magnetic source imaging approach. Cerebral Cortex, 10, 809-816.

Simões, G. C. (2012). Fluência na leitura oral e a sua relação com o conhecimento do vocabulário em leitores adolescentes (Dissertação de Mestrado, Universidade de Lisboa, Faculdade de Psicologia). Retirado de http://hdl.handle.net/10451/8070

Simos, P. G., Breier, J. I., Fletcher, J. M., Foorman, B. R., Castillo, E. M., \& Papanicolaou, A. C. (2002). Brain mechanisms for reading words and pseudowords: An integrated approach. Cerebral Cortex, 12, 297-305.

Sindelar, P. T., Lane, H. B., Pullen, P. C., \& Hudson, R. F. (2002). Remedial interventions for students with reading decoding problems. In M. R. Shinn, H. M. Walker, \& G. Stoner (Eds.), Interventions for academic and behavior problems II: Preventive and remedial approaches (pp. 703-729). Bethesda, MD: NASP.

Smith, F. (1975). The role of prediction in reading. Elementary English, 52, 305-311.

Smith, F. (1979). Reading without nonsense. New York: Teachers College Press.

Snow, C. E., Burns, M. S., \& Griffin, P. (1998). Preventing reading difficulties in young children. Washington, DC: National Academy Press.

Stanovich, K. E. (1996). Word recognition: Changing perspectives. In R. Barr, Michael L.Kamil, Peter Mosenthal, \& P. David Pearson (Eds.), Handbook of reading research (Vol. II, pp. 418-452). New Jersey: Erlbaum.

Stanovich, K. E. (2000). Progress in understanding reading: Scientific foundations and new frontiers. New York: Guilford Press.

Stanovich, K. E., \& Cunningham, A. (1984). Relation between early reading acquisition and word decoding with and without context: A longitudinal study of first-grade children. Journal of Educational Psychology, 76, 330-341. 
Sucena, A., \& Castro, S. L. (2008). Estratégias fonológicas e ortográficas na aprendizagem da leitura do Português europeu. Iberpsicología: Revista Electrónica de la Federación Española de Asociaciones de Psicología, 10 (3.16). Retirado de http://hdl.handle.net/10216/5430

Sucena, A., Castro, S. L., \& Seymour, P. (2009). Developmental dyslexia in an orthography of intermediate depth: The case of European Portuguese. Reading and Writing, 22, 791-810. doi:10.1007/s11145-008-9156-4

Swanson, H. L., \& Alexander, J. E. (1997). Cognitives processes as predictors of word recognition and reading comprehension in learning-disabled and skilled readers: Revisiting the specificity hypothesis. Journal of Educational Psychology, 89, 128-158.

Taraban, R., \& McClelland, J. L. (1987). Conspiracy effects in word pronunciation. Journal of Memory and Language, 26, 608-631.

Tellings, A., \& Bouts, L. (2011). Dutch elementary school children's attribution of meaning to written pseudowords. Reading and Writing 24, 801-812.

Thomson, B., Crewther, D., \& Crewther, S. (2006). Wots that werd? Pseudowords (non-words) may be a misleading measure of phonological skills in young learner readers. Dyslexia, 12, 289-299.

Torgesen, J. K. (1998). Catch them before they fall: Identification and assessment to prevent reading failure in young children. American Educator, 22(1-2), 32-39.

Torgesen, J. K., Rashotte, C. A., \& Alexander, A. W. (2001). Principles of fluency instruction in reading: Relationships with established empirical outcomes. In M. Wollf (Ed.), Dyslexia, fluency, and the brain. Timodium, MD: York Press.

Waters, G. S., \& Seidenberg, M. S. (1985). Spelling-sound effects in reading: Time-course and decision criteria. Memory \& Cognition, 13, 557-572.

Weekes, B. S. (1997). Differential effects of number of letters on word and nonword naming latency. Quarterly Journal of Experimental Psychology, 50, 439-456.

Whitehurst, G. J., \& Lonigan, C. J. (2001). Emergent literacy: Development from prereaders to readers. In S. B. Neuman \& D. K. Dickinson (Eds.), Handbook of Early Literacy Research (pp. 11-29). New York: Guilford Press.

Zabell, C., \& Everatt, J. (2002). Surface and phonological subtypes of adult developmental dyslexia. Dyslexia, 8, $160-177$. 